\title{
Automated ECG Analysis for Localizing Thrombus in Culprit Artery Using Rule Based Information Fuzzy Network
}

\author{
C K Roopa*, B S Harish \\ JSS Research Foundation, JSS TI Campus, Mysuru, Karnataka State (India) \\ JSS Science and Technology University, JSS TI Campus, Mysuru, Karnataka State (India) \\ Received 28 September 2018 | Accepted 15 February 2019 | Published 25 February 2019
}

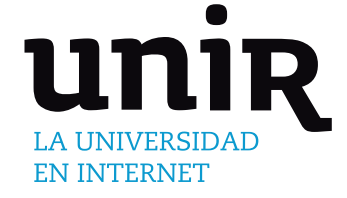

KEYWORDS

Cardio-Vascular

Diseases, ECG,

Identification,

Classification,

Information Fuzzy

Network.

OI: $10.9781 /$ ijimai.2019.02.001

\section{INTRODUCTION}

$\mathrm{T}$ HE regular 12-lead Electrocardiogram (ECG) has been employed clinically for more than 100 years for finding heart diseases [1]. An ECG supervising system evaluates the ECG signals from leads incorporated in different anatomical locations of the body for evidences of ST elevation [2]. ST elevation and depression measurements from the leads are plotted in a graphical display arranged in relation to the anatomical points that are the basis of the lead signals [3]. Supervising ECG for ST segment variation is the only non-invasive procedure that facilitates the recognition and documentation of all episodes of Ischemia as replicated by ST segment changes [4].

Machine learning techniques are being increasingly adopted for automatic identification of blocks by analyzing ECG signals. NeuroFuzzy methods can be used to estimate the unknown noise, present in the Electrocardiogram signals [5]. Adaptive neural combined with the Fuzzy System, is used to construct a fuzzy predictor for ECG analysis. For this system, setting up parameters such as the number of Membership Functions (MF), training time period and learning algorithm are determined by the learning data [6]. Due to the altered form of ECG signals with several frequency noises and existence of several arrhythmic events in a cardiac rhythm, automated elucidation of abnormal ECG classification becomes a demanding task [7].

However, adequate Identification of Thrombus in the Culprit Artery (ITCA) in the patient before performing an angiogram is necessary for

\footnotetext{
* Corresponding author.

E-mail address: ckr@sjce.ac.in
}

successful surgical outcome. Before designing an automatic thrombus identification system, the characteristics and properties of ECG signal have to be studied with respect to the thrombus location. Due to a wide range of patients with Myocardial Infarction (MI), the characteristic of ECG signal may vary among patients with the same heart disorder. Hence the main objectives of this paper includes: 1. Differentiating Non-Ischemic cause of ST elevation from Ischemic cause 2. Localizing culprit vessels and its segments by ECG using ST elevation myocardial infarction. Judgment of accurate location of a lesion in the coronary artery by analyzing the ECG signal is important in a typical intensive care or operating room for decision-making and further treatment of the patient. The key idea of the proposed method is to identify the culprit artery and thrombus location by eliminating other chances or causes such as Tachycardia using ST elevation in myocardial disorder. For this purpose, this article proposes a novel classification method for detecting the block in coronary artery, based on traditional conditions and criteria [9]. These conditions depend on ST Elevation or depression voltage levels in different lead systems and time intervals between points. The main advantage of the proposed method is that the cardiologist can make a quick decision to take further clinical procedures and can act as an aid for the physician (who is not specialized in cardiology) in primary health care centers.

The basic outline of the proposed methodology for detection of thrombus is as follows: ECG signal from the database is fed to the Savitzky-Golay filter [8] for smoothing. The feature parameters required to classify are measured using Stockwell Transform (starting point, peak point, end point), Nearest- Neighbor Interpolation (time interval between points) and measurement of peak amplitude (peak amplitude). The design of the overall recommended methodology is 
presented in Fig. 1.

Stockwell Transform [37] is an improvisation and combination of Short-Time Fourier Transform (STFT) and wavelet transform. The main function of this method is to analyze time-frequency and this paper implements Stockwell transform for point identification in ECG signal to locate the starting point, peak point, and end point of the ECG signal. Further, Nearest-Neighbor Interpolation is used for interpolating functions of more than one variable that constructs original data within the series of distinct set of identified data points. Further, it is used for calculating the time interval between two identified points in the signal. Nearest-Neighbor Interpolation is followed by Peak Amplitude Measurement, which is used to calculate the $\mathrm{mV}$ range of the base to peak voltage level. Finally, the features identified in ECG signal are classified based on the traditional criteria [9] using the Information Fuzzy Network.

In summary the proposed method consists of five steps: (i) Discretization and point detection using Stockwell Transform, (ii) Feature identification based on Nearest-Neighbor Interpolation and peak amplitude measurement, (iii) Evaluation of association rules based on traditional criteria, (iv) Classification using Information Fuzzy Network and (v) Block detection.

The organization of the article is structured as follows: Section II presents some of the current research work done in the automated localization of thrombus by classification of ECG Signal. The proposed methodology is presented in section III. The empirical results and the performance assessment of the proposed method with other traditional methods are presented in section IV, followed by conclusions in section V.

\section{LITERATURE REVIEW}

Identification of Myocardial Infarction (MI) and Ischemic beat is done on pre-processed ECG signal. Pre-processing includes removing of Baseline Wander (BW) and Noise. The basic method used for BW removal is the Butterworth filter in $[10,11]$ and the authors in $[12]$ used cut-off frequency depending on heart beat for 3rd order Butterworth filter. In [13] $0.3 \mathrm{~Hz}$ cut-off frequency and in [14] authors used a cutoff frequency of $0.5 \mathrm{~Hz}$. In [15], BW was eliminated using the low frequencies derivative-based filter. On the other hand $[16,17]$ employed moving average filtering to eliminate the BW. Further studies proposed in [30] employed median filter to estimate BW. In [25], median filter was used to remove BW by taking the difference between the residue component of Empirical Mode Decomposition (EMD) and Original signal. Discrete Wavelet Transform (DWT) has also been employed for BW elimination. Many techniques are employed in literature to lessen the noise in ECG signals. Low-pass filters are the simplest once. Authors in [15] applied low-pass filters to remove high frequency noise. Author in [8] used SG filter with order 5. While in [26] author used Butterworth filter without specifying frequency and order of the filter. Low-pass filtering is also been employed using moving average filters in $[19,26]$. After pre-processing the ECG signals, feature extraction is done to find the MI and Ischemia.

The feature extraction method uses the amplitude, points, time interval, segments and frequencies of the ECG. The Fourier transform is the most important method in signal processing which signifies the quantity of the signal made up by each frequency. Similarly Wavelet Transform, Fourier Transform, Principal Component Analysis (PCA) and reconstructed phase space (RPS) theory can be used in feature extraction [47]. Features extraction from wavelet transform, wavelet $\mathrm{db} 4$ [31], db6 [30] and db9 [19] is also useful in the identification of MI. EMD is used for extraction of feature in [31]. Since EMD can decompose signal into different frequencies, only 2 nd level component are used. However, the first level corresponds to noise.

In literature many methods have been employed for dimensionality reduction for MI detection. Forward Feature Selection (FFS) used in $[32,35]$ is computationally more efficient over simple filer based methods. 840 features composed of wavelet coefficients was reduced using Fuzzy rough feature selection in [44] using different level of wavelets. Particle Swarm Optimization (PSO) [32] and Bat algorithm [27] have been used in features selection. Machine learning algorithms like Random Forest and Lasso are also used in features selection. In [36], Multilayer Perceptron (MLP) eliminates irrelevant features for classification.

Selected features are used in majority of classification techniques as normal or $\mathrm{MI} /$ ischemia ECG signal. Thresholding method was widely used for ECG classification in [20, 22, 45, 28, 29]. Using K value as $5, \mathrm{KNN}$ classifier was used in [18] to classify the signal using description found in [46]. Support Vector Machines (SVMs) have been widely used for classification of ischemia and MI with linear [17, 18, 24, 33], Radial Basis Function (RBF) [18, 21, 25, 35], polynomial [11] and exponential chi-squared kernel functions [11]. Review in [47] used artificial neural networks, fuzzy logic, rough set theory, decision trees, genetic and hybrid algorithms for classification of various heart

Feature Identification and Extraction

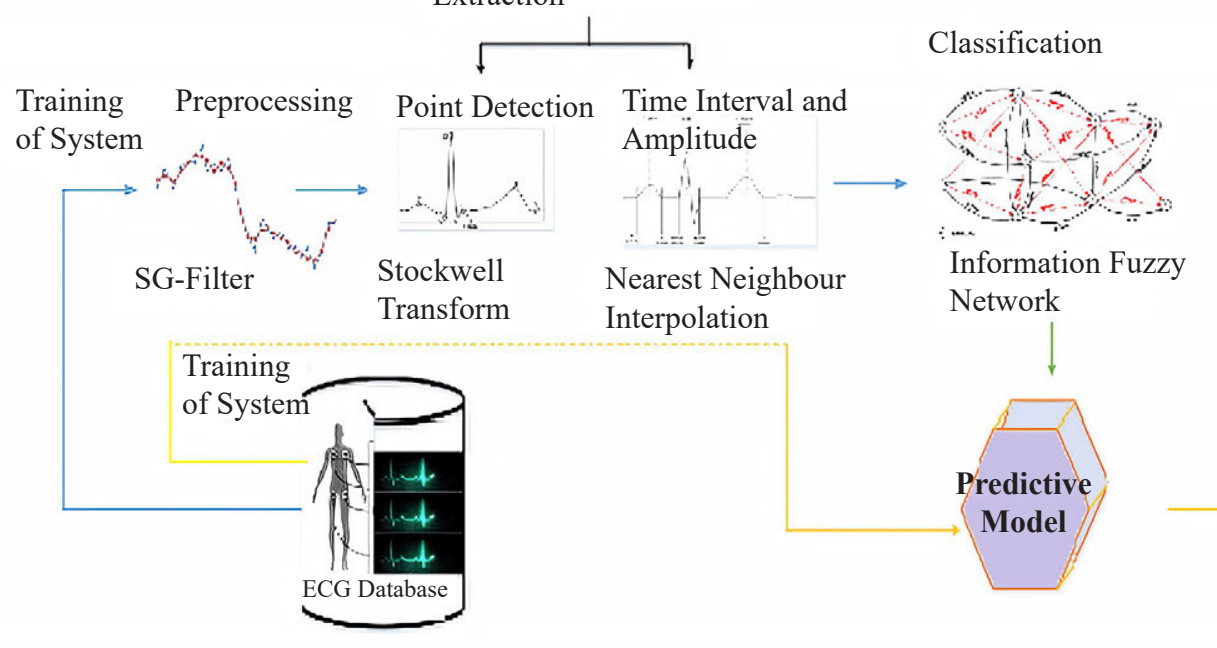

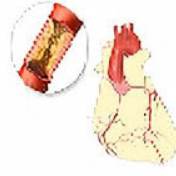

Detection of Block

Fig. 1. Location of Thrombus in the Culprit Artery using Information Fuzzy Network. 
diseases. Classifications based on Predictive Association Rules (CPAR) algorithm applied 6 association rules presented in [23] to classify ECG features. Expert knowledge is additionally been modelled with fuzzy logic in [5].

The vital role of a cardiologist is to take care of cardiac patients with utmost concerns. Thus computer aided feature extraction and analysis of ECG signals for disease identification has become a necessity. The initial step in Computer Aided Diagnosis (CAD) is the identification and extraction of the features of the ECG signals.

\section{RESEARCH Methodology}

The first stage in developing an automated ECG classifier and thrombus location is to extract the characteristic features such as the points $(\mathrm{P}, \mathrm{Q}, \mathrm{R}, \mathrm{S}$, and $\mathrm{T})$, time interval and amplitude from the waveform. Time interval and amplitude is considered as the first order and hence can be derived using Nearest-Neighbor Interpolation and Amplitude measurement technique. On the other hand, point detection can be obtained using Stockwell Transform. In a typical supervised classification scheme, the features are labeled with the decision outcome. Further, the IFN classifier is applied to the data through training and testing process.

\section{A. Identification and Extraction of Time Interval and Amplitude}

In the proposed methodology, classification of the ECG signal and identification of the culprit artery with the location of thrombus is fundamentally based on some of the features obtained from the ECG signal. The features that are identified and extracted are the point's identification (starting point, peak point, end point) of $\mathrm{P}, \mathrm{Q}, \mathrm{R}, \mathrm{S}, \mathrm{T}$, time interval between points and peak amplitude. For point identification, an ECG signal has to be converted into its time-frequency distribution and frequency-dependent resolution, while preserving a direct relationship with time spectrum component. For this purpose, the Stockwell Transform [37], is used. The advantage of using Stockwell Transform is that it gives an accurate Time-Frequency representation and gives information about all points present in the signal. After identifying the ECG points, the necessary time interval between the points are calculated using Nearest-Neighbor Linear Interpolation method. It performs linear polynomials to construct original data points within the series of distinct set of identified data points. Finally, the amplitude of the ECG points such as R peak amplitude and ST segment amplitude is calculated by implementing Amplitude Measurement technique [38], which uses uneven threshold process to identify the R-peak and ST segment amplitude.

\section{B. Stockwell Transform}

The Stockwell Transform provides a frequency dependant resolution spectrum. The advantage of this transform is that it maintains a straight association with the Fourier spectrum. In this work, the advantages of Stockwell Transform to identify the ECG points are exploited. Stockwell Transform is equivalent to applying several Short-Time Fourier Transform (STFT) with different sized windows. It can be examined as a frequency dependent STFT or a phase corrected wavelet transform. In addition, it permits accessing any frequency component in the time-frequency domain.

Once the start point, peak point and end point of $\mathrm{p}$ wave, QRS complex, ST segment and T wave are detected from each lead, there is a need to calculate the time interval between every point. Each point in an ECG signal is located with respect to its value of amplitude or voltage in Y-axis and time value in X-axis. Earlier, scaled ECG papers were used to represent the ECG signal, where each unit box represented 40 milliseconds of time on the $\mathrm{x}$-axis and $0.1 \mathrm{mv}$ on the y-axis. During digitalization, the analog signal is converted into digital, which is mapped on to a scaled function similar to that of the scaled ECG paper to identify the points present in the ECG. Further we implemented the Nearest-Neighbor Interpolation method to calculate the time interval between points.

\section{Nearest-Neighbor Interpolation}

A normal ECG signal composes of $\mathrm{P}$ wave, which is less than $0.25 \mathrm{mV}$ in amplitude and less than $0.12 \mathrm{sec}$ in width. Q wave is less than $0.04 \mathrm{sec}$ in duration and less than $25 \%$ of $\mathrm{R}$ wave, where $\mathrm{R}$ wave has amplitude of at least $0.5 \mathrm{mV}$ in limb leads and $1 \mathrm{mV}$ in pericardial leads. The $\mathrm{S}$ wave amplitude is greater than $\mathrm{R}$ wave in lead V1 and smaller than $\mathrm{R}$ wave in lead V6. $\mathrm{T}$ wave does not exceed $0.5 \mathrm{mV}$ in limb leads and it is $1 \mathrm{mV}$ in pericardial leads. Also, normal PR interval ranges between 0.12 and $0.20 \mathrm{sec}, \mathrm{QRS}$ duration ranges between 0.07 to $0.10 \mathrm{sec}$, QT interval ranges between 0.33 to $0.43 \mathrm{sec}$ and ST interval is 0.30 to $0.34 \mathrm{sec}$. In order to identify the points in the ECG we make use of the Nearest-Neighbor interpolation. By definition, NearestNeighbor interpolation is a process of determining approximate values of a function within a range bounded by two function values. In ECG signal analysis, once the points are detected, the interval between these points is determined using the Nearest-Neighbor interpolation method.

In this research work, we adopted a variable threshold method for peak amplitude measurement. We need to measure the amplitude of each segment, particularly R point and the peak of ST segment with $\mathrm{J}$ point. Since, we know the amplitude of every point on normal ECG signal, $\mathrm{P}$ wave which is less than $0.25 \mathrm{mV}$, Q wave has amplitude of $0.30-\mathrm{mV}$, R wave has amplitude of $1.5 \mathrm{mV}$, S wave has amplitude of $0.40-\mathrm{mV}$ and $\mathrm{T}$ wave does not exceed $0.5 \mathrm{mV}$. An amplitude threshold is determined as a fraction of the largest positive valued factor of that array. The threshold is fixed by defining $d_{t h}$ for each ECG signal, which is calculated using the following equation,

$$
d_{t h}=\frac{1}{2}((0.5 \times \max (\operatorname{point}[q])+\operatorname{mean}(\operatorname{point}[q]))
$$

The peak amplitude measurement is greatly affected by baseline wandering, which is the instability of the base line or change in isoelectric line position, which is likely to be moving up or down. This is primarily caused by improper connections in the ECG leads. Filtering the baseline can reduce wandering, thereby increasing the accuracy of amplitude measurement.

Fig. 2 shows all the features extracted (amplitude and time interval of each wave (P, Q, R, S, T, and U)) as required for classification and block localization in the culprit artery.

P wave threshold is $0.20 \mathrm{mV}$, Q wave threshold is $-0.15 \mathrm{mV}, \mathrm{R}$ wave threshold is $0.9 \mathrm{mV}, \mathrm{S}$ wave threshold is $-0.40 \mathrm{mV}$ and $\mathrm{T}$ wave does not exceed $0.5 \mathrm{mV}$. When the peak is above the threshold, it is detected successfully. The entire feature identification and extraction process is presented in Fig. 2. Classification of ECG signal for locating block in the culprit artery is the next stage and it is presented in the next section.

\section{Classification of ECG Signal}

Several techniques for detecting blocks in coronary artery using ST segment in ECG signals have been analyzed. At present, medical equipment can vigilant a physician to the probable changes in ST and $\mathrm{T}$ wave deviations. But a specialist must still inspect these changes to decide whether they are due to blockage or not. Present methods of automatic classification for location of blocks are time-consuming and cannot discover blocks in real-time. This motivates a need for a capable system for automatic block detection. ECG Signal classification based on Information Fuzzy Network (INF) with rule based criteria distinguishes between different ECG signals that have different characteristics based on the extracted features analyzed earlier in this paper. 


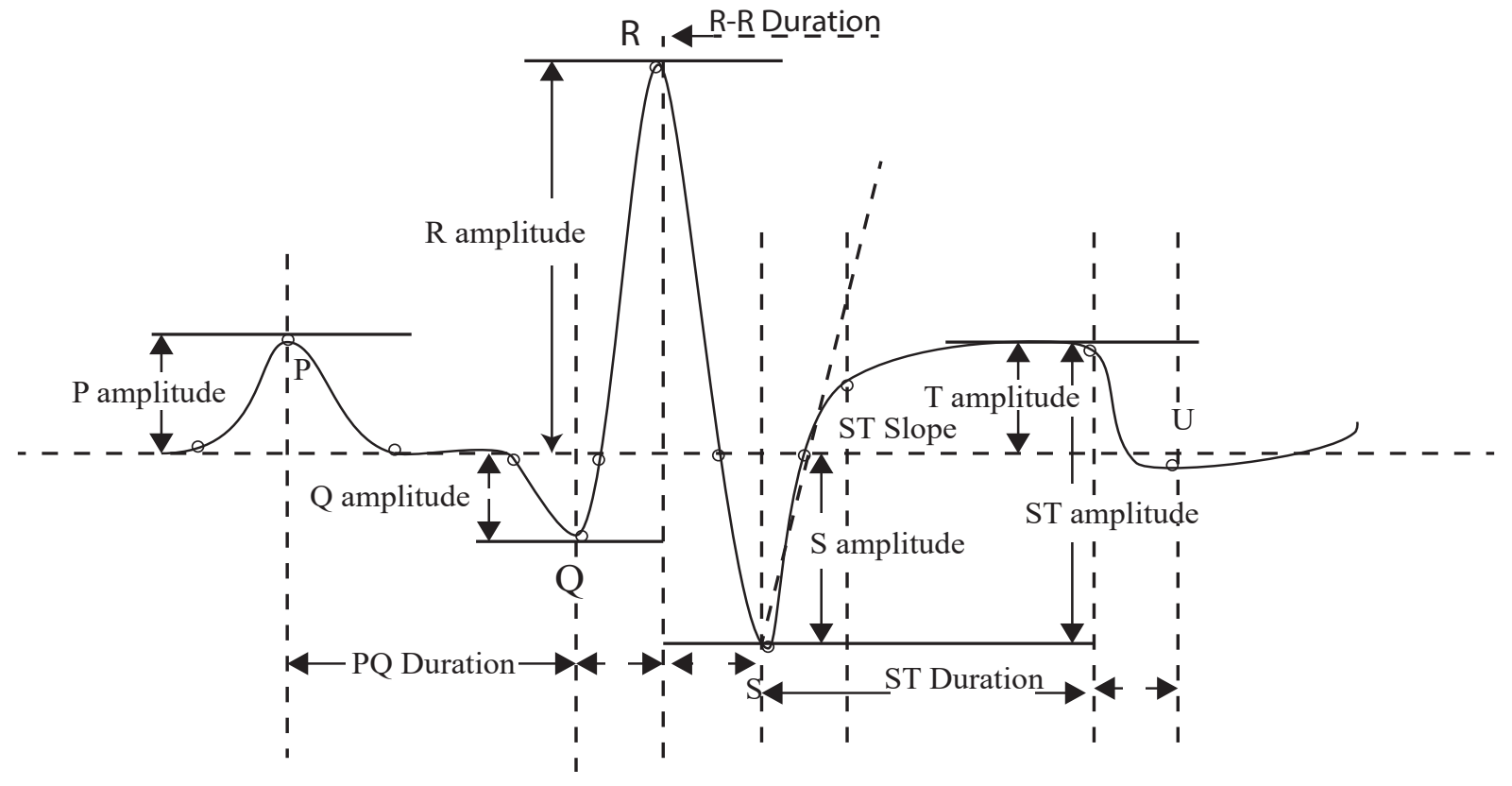

QR Duration RS Duration

TU Duration

Fig.. 2. Extracted features from one ECG cycle.

\section{Differentiating Ischemia and Non-Ischemic ECG Signals}

An easy to-use, simple and accurate classification for locating the block has been proposed based on the evaluation of ST-segment changes in a 12- lead ECG that predicts the site of block in patients. There are several techniques employed for detecting Ischemia. However there are two methods which are widely used: coronary angiography and echocardiogram. While angiography is an invasive method for detection with $98 \%$ accuracy, echocardiogram is a noninvasive method with an accuracy of around $85 \%$.

A block in the coronary artery causes variations in the depolarization of the heart's ventricular tissue. If the cardiac tissue is Ischemic, it results in improper depolarization. This shows an improper variation in current pulse, which causes a depression in the T-Q segment and an elevation in the ST segment. ST deviation can be calculated by using equations (2), (3) and (4) as follows,

To find ST Depression,

$$
P R \operatorname{point}\left(i^{\prime} j\right)=\frac{1}{2} \operatorname{ceil}\left(R \operatorname{loc}(i, j)-\left(S_{o f f}(i, j)-Q_{o n}(i, j)\right)\right.
$$

To find ST Elevation,

$$
S T \text { po } \operatorname{int}(i, j)=\frac{1}{2} \text { ceil }\left(\text { Tloc }(i, j)-T_{o f f}(i, j)-T_{o n}(i, j)\right)
$$

To find ST Deviation,

$$
S T \text { Deviation }(i, j)=a b s(x(P R \text { po } \operatorname{int}(i, j), i)-x(S T \text { point }(i, j), i))
$$

Where ceil is the Ceiling Function, the least integer greater than or equal to $x$ abs is the Absolute Value, indicating the distance of the point or number from the zero point; the value of $T_{\text {on }}$ and $S_{\text {off }}$ is the first offset of $S$ signal and $T_{\text {on }}$ is the first onset of T wave which separates ST segment. From peak detection, zero crossing point behind the peak is considered as onset and zero crossing point ahead of the peak is detected as offset.

The ST deviation thus obtained is divided by 100; a normal ST deviation would be less than 0.148. Ischemia is detected if the ST Deviation obtained is greater than 1 (these condition are fixed using cardiologist input). We confirmed that the ST deviation is caused by a block in coronary artery and if the condition is false, the ST deviation may be due to Non-ischemic causes. The flowchart given in Fig. 3 illustrates the method of differentiating Ischemia and Non-Ischemia.

\section{Rule based Criterions for identifying the Culprit Artery and Localizing the Thrombus}

One of the main objectives of this article is to identify the culprit artery from Ischemic ECG signals and finally to locate the exact position of the block as proximal, middle or distal. For this purpose, the classification process depends on the conditions given by [39]. The conditions for predicting the culprit artery is obtained from practical cases in real time situations. However, the main issue with this is the fact that the conditions are derived from independent leads. To overcome this problem, we adopted an ECG lead selector for analyzing the corresponding lead. The major conditions for identifying the culprit artery are thus: if ST elevation in leads L2, L3, aVF (limb lead), and L2 elevations are greater than L3 elevation, it is detected as Left Circumflex Artery (LCx). If L3 elevation is greater than L2 elevation, then it is detected as Right Coronary Artery (RCA). One more condition for RCA is that the V1 lead (Chest lead) must be greater than the V3 lead. Similarly, if V3 elevation is greater than the V1 lead, then it is predicted as the Left Anterior Descending Artery (LAD).

Further identifying the culprit artery, the exact spot of the block needs to be localized. Since identification in LAD is the lengthiest process as compared to the other branches, locating the block in LAD is valuable. Hence LAD is considered for locating the block. The primary condition is the presence of ST deviation in the lead aVF, L2 and L3, it is then predicted as the Proximal to major septal artery LAD. The next condition is the ST elevation in L1, avl, V5, V6 and it is then a proximal to major diagonal artery of LAD. Similarly if ST elevation is in L2, L3 and aVF, then it is distal to major septal artery of LAD. Finally ST elevation in V2, V3, and V4 with Right Bundle Branch Block (RBBB) is predicted as Proximal LAD. The remaining sub conditions for identification and localization are depicted in Fig. 4 and Fig. 5. 


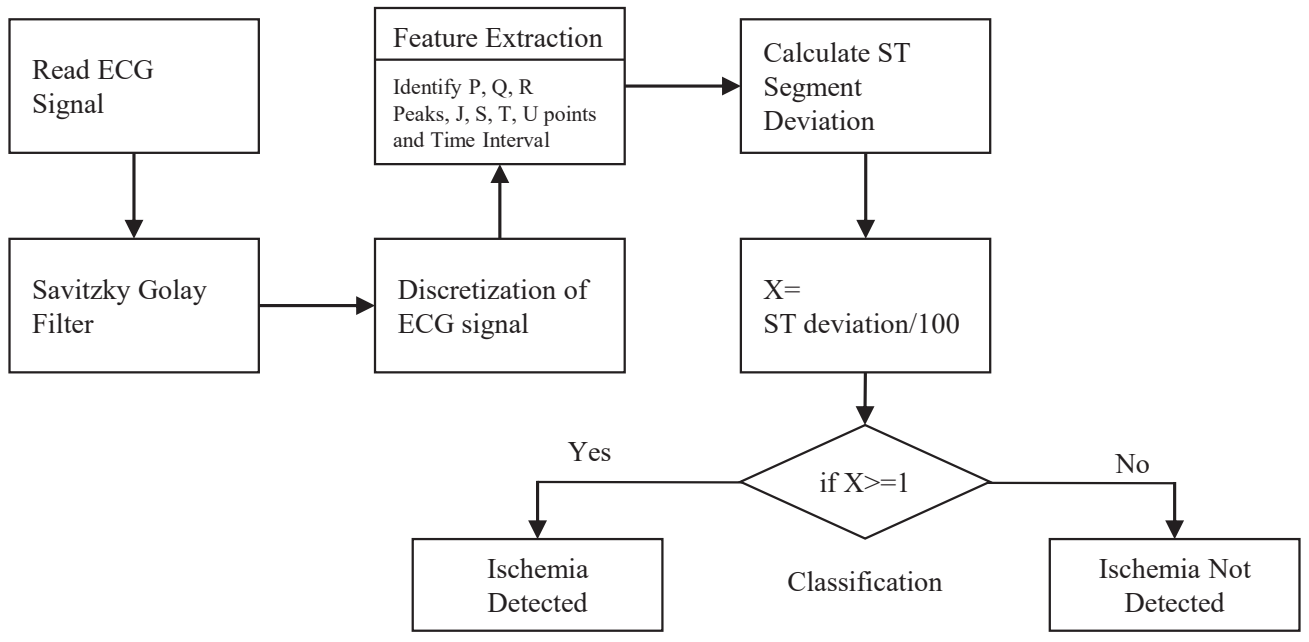

Fig. 3. Flow chart for differentiating Ischemia and Non-Ischemic.

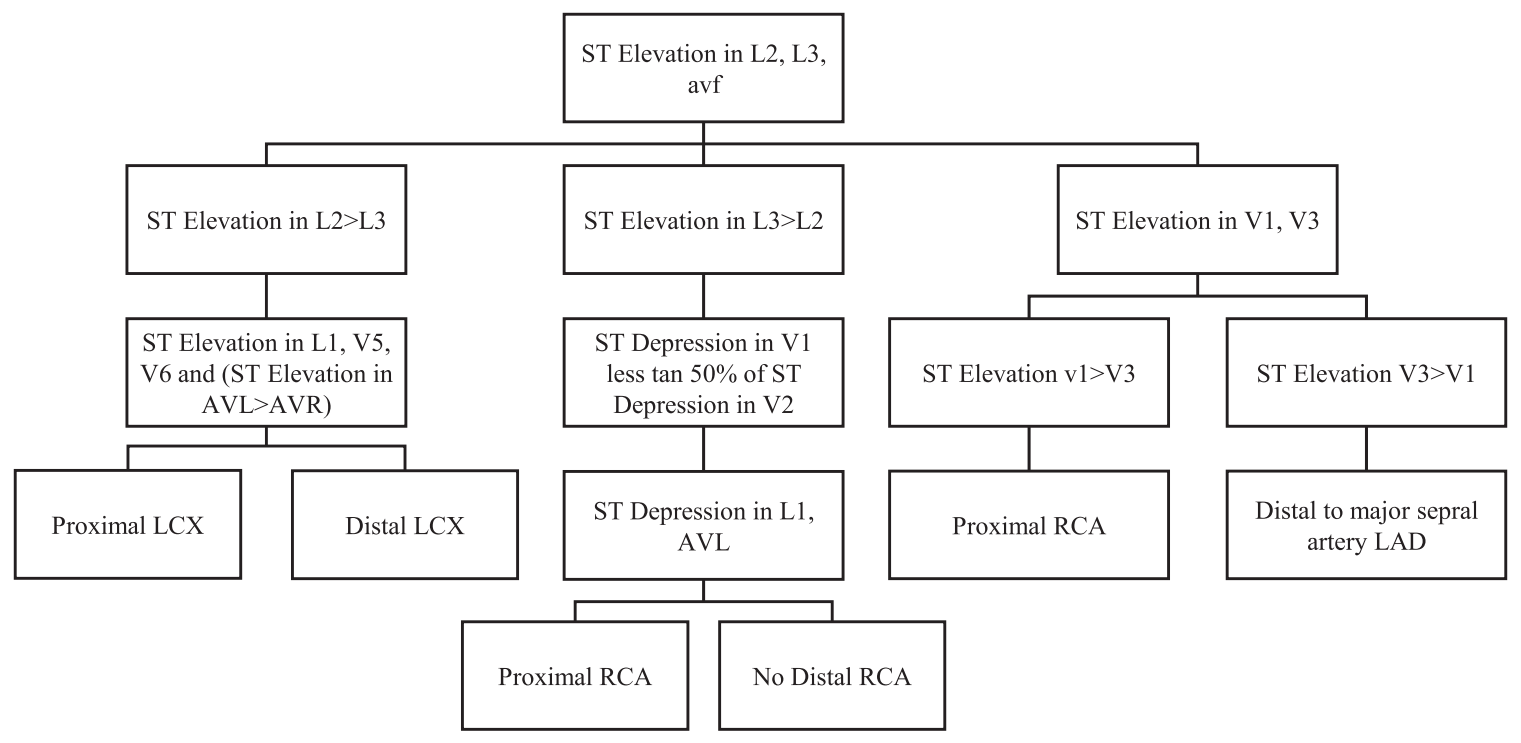

Fig. 4. Flow chart to identify the Culprit Artery.

\section{E. Information Fuzzy Network for ECG Classification and} Identification of Block

The proposed approach for ECG classification and block location is based on the Information-Fuzzy Network (IFN). The IFN produces a ranked list of outputs relevant to a given set of input features as a result of inducing a correct classification task. Fig. 6 shows the model of the information fuzzy network.

IFN is a greedy machine-learning algorithm for supervised learning. IFN consists of many nodes. Every node inside the net is called as the inner or hidden node. Each node is weighted with conditional mutual information. IFN uses the conditional information in order to classify features during the identification stage. The set of nodes in the column are called layers, and each layer carries a condition for feature classification. However, there cannot be more than one condition in a layer. The IFN model is typically more stable, which means that small variations in the training set will affect it less, when compared to other models.

The whole classification process is made functional by imposing the previous conditions defined over the IFN. The root node is associated with the features that are extracted during the feature identification and extraction stage (such as amplitude and time interval). Each hidden layer consists of nodes representing equivalence classes of the corresponding input variable. There are three hidden layers other than the root layer. Root layer is considered as the layer 0 , while layer 1 , layer 2 and layer 3 are the hidden layers and the target layer is the output layer. Layer 1 has the attributes or conditions for the Ischemic or Non-Ischemic causes. Layer 2 consists of two classes as shown in Fig. 6 . The first class has the attributes or conditions for the classification of supraventricular from ventricular tachycardia, while the second class has the attributes or conditions for identifying the culprit artery. The IFN automatically determines the equivalence classes as contiguous sub-ranges of input values. Finally, the output layer has five output variables that indicate the location of the block in the culprit artery.

\section{Training IFN}

A training set is a set of data employed to uncover the potentially predictive relationships between the input and the output. A test set is a set of data used to evaluate the strength and effectiveness of a prognostic association. Test and training sets are used in IFN, so that it can make predictions on new data. The accuracy and reliability of the block detection depends on the type of training set used. The database from MIT Physionet databank is divided into two splits: $30 \%$ of data to train and $70 \%$ to test the system.

Training phase is also called as weight updating or tuning phase 


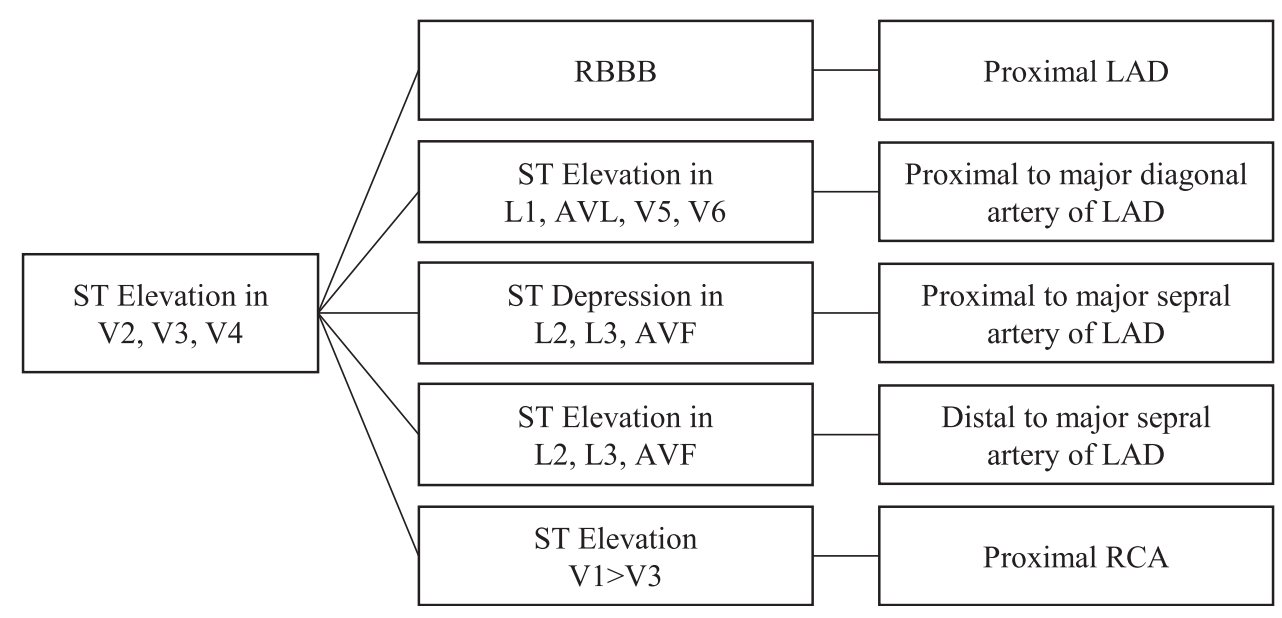

Fig. 5. Flow chart for localizing the Thrombus.

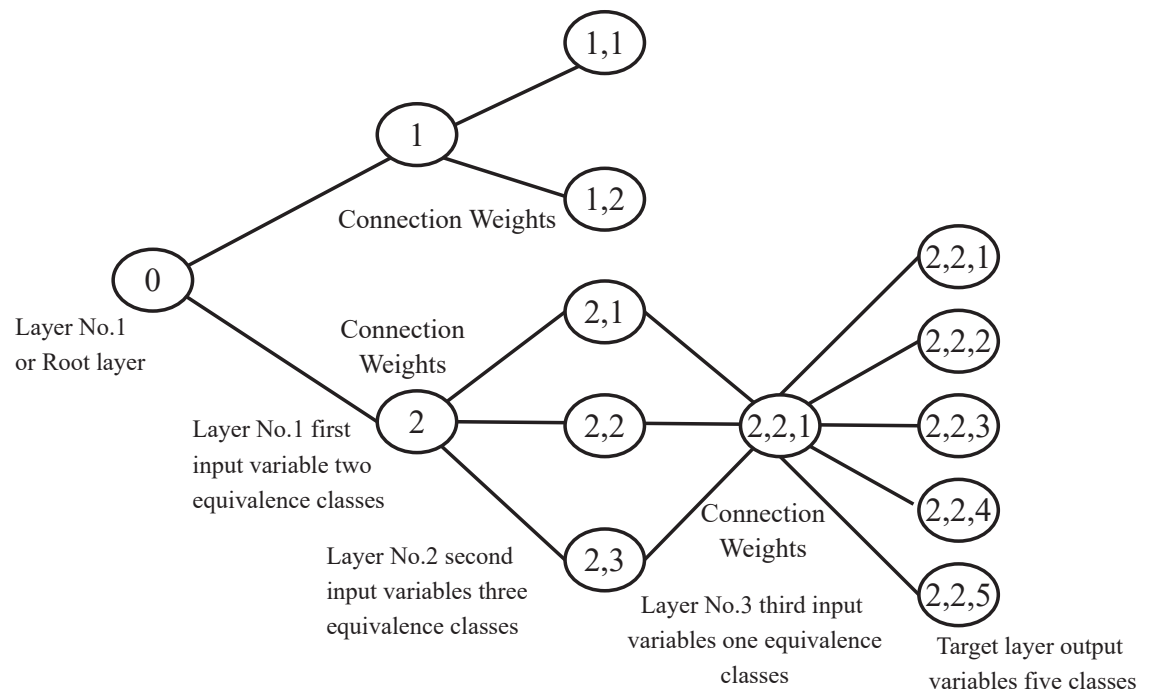

Fig. 6. IFN model for ECG classification and identification of block using Culprit Artery.

of the system. During training, the ECG signal from the database is applied to the system and the corresponding output is analyzed for error function. The objective of the training phase is to reduce the error function so that the result obtained from testing would be more accurate. The training error of the estimated conditional mutual information between an input attribute $\mathrm{Ai}$ and the target attribute $\mathrm{Bi}$ in training phase is evaluated by using the likelihood-ratio statistic. The likelihood-ratio test is a general-purpose technique for testing the null hypothesis Ho, that two random variables are statistically independent. This likelihood-ratio can be used to compute a p-value or compared to a critical value to decide whether to reject the null model in favor of the alternative model. The default significance level ( $\mathrm{p}$-value) used by the IFN algorithm is $0.1 \%$. Likelihood-ratio statistic is given by,

$$
R^{2}\left(A_{i} ; B_{i} / n\right)=2 \bullet(\operatorname{In} 2) \bullet E^{*}(n) \bullet M I\left(A_{i} ; B_{i} / n\right)
$$

In the above equation (5) $E^{*}(n)$ is the number of cases correlated with the node $n$. After detecting the training phase error, it needs to be minimized by adjusting the weighted function of IFN. The weights are modified in proportion to their contribution to the error. Updating is done after each cycle using the conditional mutual information as mentioned in the learning section. It must be pointed out that the weights (W) are adjusted to ensure that $-0.1 \leq W \leq 0.1$ for each revised step, so that the chances of overshooting of the weights may be minimized, in the course of smoothly approaching a minimum error solution.

\section{Testing IFN}

Testing is done by computing the features over a new signal sample and choosing the class label corresponding to the IFN with the highest output. Subsequent to training, the recommended model encodes in its weights information regarding class discrimination in the feature space called label, which is the desired Information Fuzzy Classifier. In the testing phase, the $70 \%$ of data is considered as input to the IFN model and its performance is evaluated. During the testing phase, the system labels the test vector in the dimensional space. The output layer yields values by indicating the training pattern in the corresponding output classes. Once the Information Fuzzy Network identifies the inputoutput relationships, testing consists of generating the test cases that represent the union of combinatorial tests for input variables included in the IFN model at each single output. The results drawn from the proposed methodology are given in the next section.

\section{RESULTS}

In this section, we present the results using the Information Fuzzy Network approach for automated block detection in the culprit artery. We perform an automated input-output analysis by training the Information Fuzzy Network. The proposed method is implemented using Intel i5 environment over a Personal Computer with 2.99 $\mathrm{GHz}$ CPU and 8GB RAM running on Windows 7 OS. The induced information fuzzy models are utilized for block detection. We evaluate 
the performance of the proposed method by comparing Sensitivity, Specificity, Positive Predictive Value and Negative Predictive Value and Classification Accuracy as evaluation metrics.

\section{A. ECG Database}

In general, classifiers with a higher number of output classes require more features, which imply the need for a larger database. However, to prevent over-training, which would mean great performance on the training set, but a poor performance in testing sets, the database considered represents a well-defined differentiating feature set. Hence, we selectively combined some of the databases including ECG signals of patient with Ischemic Myocardial Infarction, Ventricular Tachycardia, Supraventricular Tachycardia and ECG signal of normal patient. The proposed methodology is implemented using MATLAB R2013a platform, and the experimental results are analyzed and compared with the conventional methods. The data chosen from MIT Physionet databank for training and testing are: Long-Term ST Database, consisting of 86 records that are 21 to 24 hours long, and contain two or three ECG signals, annotated beat-by-beat. With respect to the ST episodes as the rhythm changes, the signal quality changes. Each record includes ST level time series based on 16-second averages centred on each beat. Spontaneous Ventricular Tachyarrhythmia Database contains 135 pairs of RR interval time series, recorded by implanted cardioverter defibrillators in 78 subjects. Each series contains between 986 and 1022 RR intervals. One series of each pair includes a spontaneous episode of Ventricular Tachycardia (VT) or Ventricular Fibrillation (VF) and the other is a sample of the intrinsic (usually sinus) rhythm. T-Wave Alternans Challenge Database contains 100, 2, 3, and 12-lead ECG records sampled at $500 \mathrm{~Hz}$ with 16-bit resolution over a $\pm 32 \mathrm{mV}$ range, including subjects with risk factors of sudden cardiac death as well as healthy controls and synthetic cases with calibrated amounts of T-wave Alternans [37]. The output ECG signals and results are illustrated in Fig. 7(a). Fig. 7(a) shows the original ECG signal from database and Fig. 7 (b) shows the filtered ECG signal using Savitzky-Golay filter.

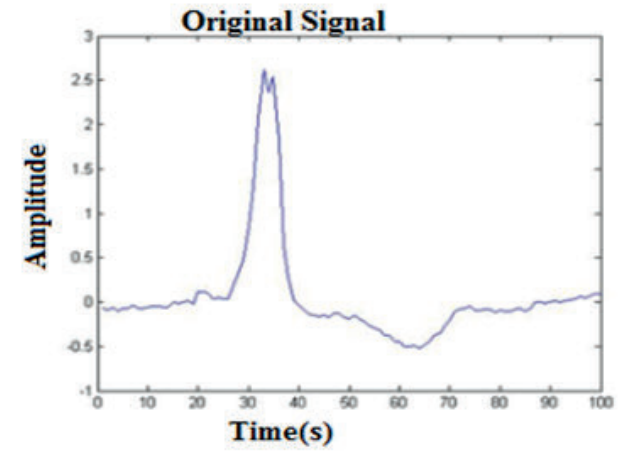

(a)

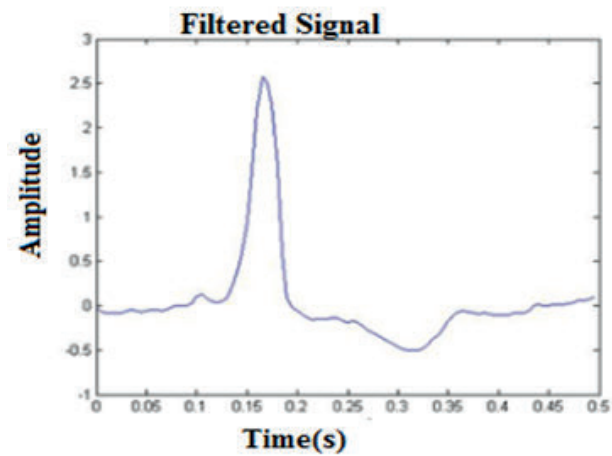

(b)

Fig. 7. (a) Original ECG signal (b) Filtered ECG signal using Savitzky-Golay filter.

\section{Thresholding Peaks in Signal}
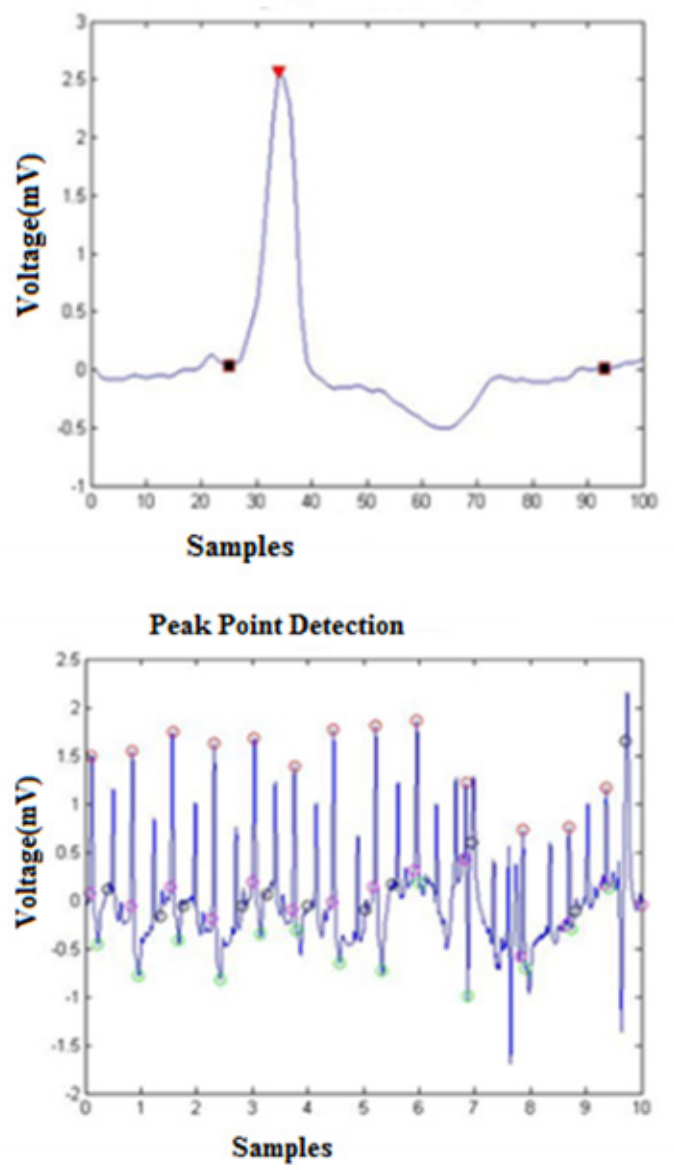

Fig. 8. Detection of points in ECG signal.

The original ECG signal shown in Fig. 7(a) is given as the input signal to the Savitzky-Golay filter. The output from the filter is further used for feature extraction process. The detection of points in the ECG signal is shown in Fig. 8. This figure shows the point detection by Stockwell transform. The extracted features such as time interval and amplitude of each point on ECG are tabulated in table I.

TABLE I. Time Interval And Amplitude Of ECG Segment

\begin{tabular}{cccc}
\hline $\begin{array}{c}\text { ECG } \\
\text { Segments }\end{array}$ & $\begin{array}{c}\text { Time } \\
\text { Interval }(\mathbf{m s})\end{array}$ & ECG points & $\begin{array}{c}\text { Amplitude } \\
(\mathbf{m V})\end{array}$ \\
\hline RS segment & 0.06271 & $\mathrm{~S}$ & 0.4590 \\
RR segment & 0.14988 & $\mathrm{~T}$ & 0.4090 \\
ST segment & 0.35 & $\mathrm{Q}$ & 0.3490 \\
\hline
\end{tabular}

Table I shows the time interval of RS segment, RR segment and ST segment and the amplitude of the ECG Signal. In order to prove the efficiency and accuracy of the proposed method, the analytical performance of the proposed block detection using IFN approach is validated by the metrics given in the next section.

\section{B. Performance Evaluation}

Performance evaluation is carried out by comparing classification accuracy of the proposed methodology with existing classifiers such as Support Vector Machine [40], Compound Neural Network [41] and Rough-Set-Based Inference Engine [42, 43] for ECG classification with five parameters: Sensitivity, Specificity, Positive Predictive Value (PPV), Negative Predictive Value (NPV) and Classification Accuracy.

The five performance parameters described above depicts the performance of the proposed methodology in terms of classification 
of ECG signal and the detection of the block. The result shows the effectiveness of the system performance (see Table II). The efficiency of the proposed method can be well shown with the aid of comparative analysis. A comparison of the proposed method with other existing methods is given in the next section.

TABle II. Performance Evaluation of the Proposed Method

\begin{tabular}{cc}
\hline Performance Parameters & $\begin{array}{c}\text { Proposed Thrombus } \\
\text { Detection using Information } \\
\text { Fuzzy Network (IFN) }\end{array}$ \\
\hline Sensitivity & $87.50 \%$ \\
Specificity & $100 \%$ \\
PPV & $100 \%$ \\
NPV & $83.33 \%$ \\
Classification Accuracy & $92.30 \%$ \\
\hline
\end{tabular}

\section{Performance Comparisons}

In this section, we compare the proposed method with Generalized Least Square-Support Vector Machine method, Compound Neural Network and Rough-Set-Based Inference Engine. In the first method, five types of ECG beats and the features from three transform approaches are independently classified using Least Square-Support Vector Machine (LS-SVM) [42].The second method is based on a Compound Neural Network (CNN) to classify ECGs as normal or carrying an Atrio-Ventricular heart Block (AVB) [41]. This method uses three different feed forward multilayer neural networks. The third method is a rule-based rough-set decision system for the development of a disease inference engine [42], [43]. The proposed system is based on the criteria from popular medical literature and feedback from reputed cardiologists. All the performance evaluation metrics are used for the comparison are tabulated in table III.

TABLE III. Comparison of the Proposed Method with other Existing Methods

\begin{tabular}{ccccc}
\hline $\begin{array}{c}\text { Generalized } \\
\text { Performance } \\
\text { Parameters } \\
\text { Support } \\
\text { Vector } \\
\text { Machine }[42]\end{array}$ & $\begin{array}{c}\text { Compound } \\
\text { Neural } \\
\text { Network } \\
{[41]}\end{array}$ & $\begin{array}{c}\text { Rough } \\
\text { Set Based } \\
\text { Inference } \\
\text { Engine } \\
{[42,43]}\end{array}$ & $\begin{array}{c}\text { Information } \\
\text { Fuzzy } \\
\text { Network (IFN) } \\
\text { (Proposed } \\
\text { Method) }\end{array}$ \\
\hline Sensitivity & 84.62 & 65.54 & 48.00 & $\mathbf{8 7 . 5 0}$ \\
Specificity & 83.33 & 92.25 & 96.00 & $\mathbf{1 0 0}$ \\
PPV & 81.48 & 63.47 & 85.30 & $\mathbf{1 0 0}$ \\
NPV & 86.21 & 92.09 & 91.70 & $\mathbf{8 3 . 3 3}$ \\
Classification & 85.59 & 87.01 & 91.00 & $\mathbf{9 2 . 3 0}$ \\
Accuracy & & & & \\
\hline
\end{tabular}

From Table III, it is clear that the ECG signal can be used as a reliable indicator for detecting heart blocks. In this research work, ECG analysis is effectively used as a non-invasive tool for the classification and location of the block in the coronary artery. Here, our proposed methodology was compared with Compound Neural Network, Rough Set based Interface Engine and Least Square-Support Vector Machine in terms of Sensitivity, Specificity, and Classification Accuracy. The obtained results proved a valid performance improvisation.

The proposed method for block detection in the culprit artery based on traditional conditions derived from real time incidents is presented. These conditions are formed as a generalized algorithm and implemented using Information Fuzzy Network. The results of the proposed method are tabulated in Table II and Table III. The results of the filtering method used are presented in Fig. 7. It shows the original ECG signal from the database and the filtered ECG signal. From Table III, it is clear that the proposed system is highly efficient, as the rate of Sensitivity is $87.5 \%$, Specificity is $100 \%$ and Classification
Accuracy is $92.30 \%$; which are more accurate as compared to other methods. Also, the rate of Positive Predictive Value is $100 \%$ and Negative Predictive Value is $83.33 \%$ and classified output is high when compared to other methods. Similarly, to prove the effectiveness of the proposed methodology, it has been compared with other conventional works that use different ECG classification to analyze ECG signal. The comparisons results presented in table 3 depicts that the results achieved by implementing the proposed method are far better than the works proposed earlier.

\section{Conclusion}

In this research work, a method for identification of culprit artery along with detection of block location based on Information Fuzzy Network using ECG signals is developed. The decision of the proposed classification results are obtained from traditional criteria. The proposed detection system is validated successfully with 264 ECG samples with ST episodes variation, RR interval time series variation and T-Wave Alternant signals from both genders and different ages. The proposed method first distinguishes the ECG signals as Ischemic or Non-Ischemic, identifies the culprit artery and finally locates the block in the identified artery. The proposed detection system achieves $92.30 \%$ of accuracy. All the input voltage parameters and the output of the logical expressions related to the proposed detection criteria are expressed in the IFN system.

In future, we are intended to apply deep networks which could further enhance the results of the proposed method. It is also possible to employ various other soft computing techniques to find the percentage of blockage in the culprit artery.

\section{REFERENCES}

[1] R. S. Bruning and M. Sturek, "Benefits of exercise training on coronary blood flow in coronary artery disease patients," Progress in cardiovascular diseases, Vol.57, No.5, 2015, pp.443-53.

[2] A. Arbab-Zadeh and V. Fuster, "The myth of the vulnerable plaque: transitioning from a focus on individual lesions to atherosclerotic disease burden for coronary artery disease risk assessment," Journal of the American College of Cardiology, Vol.6, No.8, 2015, pp.846-855.

[3] P. Guzik and M. Malik, "ECG by mobile technologies," Journal of electrocardiology, Vol.49, No.6, 2016, pp.894-901.

[4] C. Haarmark, R. P. Hansen, E. Vedel-Larsen, S. H. Pedersen, C. Graff, P. Mads Andersen, M.D. Egon Toft, F. Wang, J. J. Struijk, MScEng, K. Jørgen and Kanters, "The prognostic value of the Tpeak-Tend interval in patients undergoing primary percutaneous coronary intervention for ST-segment elevation myocardial infarction," Elsevier, Journal of Electrocardiology, Vol.42, 2009, pp.555- 560.

[5] S. Ansari, N. Farzaneh , M. Duda, K. Horan and H. Andersson, "A Review of Automated Methods for Detection of Myocardial Ischemia and Infarction using Electrocardiogram and Electronic Health Record," IEEE Reviews in Biomedical Engineering, Vol. 10, 2017, pp. 264-297.

[6] G. Stephen Ellis, M. Tendera, A. Mark de Belder, J. Ad van Boven, P. Widimsky, L. Janssens, H.R. Andersen, A. Betriu, S. Savonitto, J. Adamus, Z. Jan Peruga, M. Kosmider, O. Katz, T. Neunteufl, J. Jorgova, M. Dorobantu, L. Grinfeld, P. Armstrong, R. Bruce Brodie, C. Howard Herrmann, G. Montalescot, F.-J. Neumann, B. Mark Effron, S. Elliot, M. Barnathan, and J. E. Topol, "Facilitated PCI in Patients with ST-Elevation Myocardial Infarction," The classification England journal of medicine, Vol.358, No.21, 2008, pp.2205-2217.

[7] O. Fröbert, B. Lagerqvist, K. Göran Olivecrona, E. Omerovic, Thorarinn Gudnason, M. Maeng, M. Aasa, O. Angerås, F. Calais, M. Danielewicz, D. Erlinge, L. Hellsten, U. Jensen, C. Agneta Johansson, A. Kåregren, J. Nilsson, L. Robertson, L. Sandhall, I. Sjögren, O. Östlund, J. Harnek, and K. Stefan James, "Thrombus Aspiration during ST-Segment Elevation Myocardial Infarction," The classification England Journal of Medicine, Vol.369, No.17, 2013, pp.001-011.

[8] C.K. Roopa and B.S. Harish, “An Empirical Evaluation of Savitzky-Golay 
(SG) Filters for Denoising ST Segment," Springer, Cognitive Computing and Information Processing, Vol.801, 2018, pp.18-28.

[9] D. Da Costa, W.J. Brady, and J. Edhouse. "Abc of clinical electrocardiography: Bradycardias and atrioventricular conduction block," BMJ: British Medical Journal, Vol. 324, No.7336, 2002, pp. 535-538.

[10] S. Padhy, L. Sharma, and S. Dandapat, "Multilead ECG data compression using SVD in multi resolution domain," Biomedical Signal Process Control, vol. 23, 2016, pp. 10-18.

[11] S. Padhy and S. Dandapat, "Third-order tensor based analysis of multilead ECG for classification of myocardial infarction," Biomedical Signal Process Control, vol. 31, 2017, pp. 71-78.

[12] J. Garcia, M. Astrom, J. Mendive, P. Laguna, and L. Sornmo, "ECG based detection of body position changes in ischemia monitoring," IEEE Transactions on Biomedical Engineering, vol. 50, no. 6, pp. 677-685, 2003.

[13] M. Schmidt, M. Baumert, A. Porta, H. Malberg, and S. Zaunseder, "Two dimensional warping for one-dimensional signals-conceptual frame work and application to ECG processing," IEEE Transactions Signal Process, vol. 62 , no. 21, 2014, pp. 5577-5588.

[14] M. Hadjem, F. Nait-Abdesselam, and A. Khokhar, "ST-segment and T wave anomalies prediction in an ECG data using RUSBoost," IEEE 18th Internatinal Conference on e-Health Networking, Application and services, 2016, pp. 1-6.

[15] H.N. Murthy and M. Meenakshi, "ANN, SVM and KNN classifiers for prognosis of cardiac ischemia-A comparison," Bonfring International Journal of Research Communication Engineering, vol.5, no. 2, 2015, pp. $7-11$.

[16] A.K. Bhoi, K.S. Sherpa, and B. Khandelwal, "Classification probability analysis for arrhythmia and ischemia using frequency domain features of QRS complex," International Journal of BioAutomation, vol. 19, no. 4, 2015, pp. 531-542.

[17] S.S. Nidhyananthan, S. Saranya, and R.S.S. Kumari, "Myocardial infarction detection and heart patient identity verification," International Conference Wireless Communication Signal Processing Network, 2016, pp. 1107-1111.

[18] L.N. Sharma, R.K. Tripathy, and S. Dandapat, "Multiscale energy and eigenspace approach to detection and localization of myocardial infarction," IEEE Transaction Biomedical Engineering, vol. 62, no. 7, 2015, pp. 1827-1837.

[19] H. Pereira and N. Daimiwal, "Analysis of features for myocardial infarction and healthy patients based on wavelet," Conference on Advances in Signal Processing, 2016, pp. 164-169.

[20] J. Garcia, P. Lander, L. Sornmo, S. Olmos, G. Wagner, and P. Laguna, "Comparative study of local and Karhunen-Loeve-based STT indexes in recordings from human subjects with induced myocardial ischemia," Computer And Biomedical Research, vol. 31, no. 4, 1998, pp. 271-292.

[21] Y.L. Tseng, K.S. Lin, and F.S. Jaw, "Comparison of support-vector machine and sparse representation using a modified rule-based method for automated myocardial ischemia detection," Computation and Mathematical Methods in Medicine, vol. 2016, 2016, pp. 1-8.

[22] F. Jager, G.B. Moody, and R.G. Mark, "Detection of transient ST segment episodes during ambulatory ECG monitoring," Computers and Biomedical Research, vol. 31, no. 5, 1998, pp. 305-322.

[23] T.P. Exarchos, C. Papaloukas, D.I. Fotiadis, and L.K. Michalis, "An association rule mining-based methodology for automated detection of ischemic ECG beats," IEEE Transaction Biomedical Engineering, vol. 53, no. 8,2006 , pp. 1531-1540.

[24] J. Park, W. Pedrycz, and M. Jeon, "Ischemia episode detection in ECG using kernel density estimation, support vector machine and feature selection," Biomedical Engineering Online, vol. 11, no. 1, 2012.

[25] J.T.Y. Weng, J.J. Lin, Y.C. Chen, and P.C. Chang, "Myocardial infarction classification by morphological feature extraction from big 12-lead ECG data," Trends and Applications in Knowledge Discovery and Data Mining, vol. 8643 .

[26] N. Safdarian, N. J. Dabanloo, and G. Attarodi, “A new pattern recognition method for detection and localization of myocardial infarction using $\mathrm{T}$ wave integral and total integral as extracted features from one cycle of ECG signal," Scientific Research Publishing, vol. 7, 2014, pp. 818-824.

[27] P. Kora and S. R. Kalva, "Improved Bat algorithm for the detection of myocardial infarction," Springer Plus, vol. 4, no. 1, 2015.
[28] C. C. Lin,W. Hu, and Y.W. Lin, "A wavelet-based high-frequency analysis of fragmented QRScomplexes in patients with myocardial infarction," Computing in Cardiology Conference, 2015, pp. 565-568.

[29] S. Banerjee and M. Mitra, "Application of cross wavelet transform for ECG pattern analysis and classification," IEEE Transaction Instrumentation and Measurment, vol. 63, no. 2, 2014, pp. 326-333.

[30] U.R. Acharya, H. Fujita, V.K.Sudrashan, S.L. Oh, M. Adam, E .W. Joel Koh, J.H. Tan, D.N Ghista, R.J. Martis, C.K. Chua, C.K. Poo and R.S. Tan, "Automated detection and localization of myocardial infarction using electrocardiogram: A comparative study of different leads," Knowledge Based System, vol. 99, 2016, pp. 146-156.

[31] U. R. Acharya, H. Fujita, V.K. Sudrashan, S. L. Oh, M. Adam, E.W. Joel Koh, J. H. Tan, D.N Ghista, R.J. Martis, C.K. Chua, C.K. Poo and R.S. Tan, "Automated characterization and classification of coronary artery disease and myocardial infarction by decomposition of ECG signals: A comparative study," Information Science, vol. 377, 2017, pp. 17-29.

[32] B. Liu, J. Liu, G. Wang, K. Huang, F. Li, Y. Zheng, Y. Luo and F. Zhou, "A novel electrocardiogram parameterization algorithm and its application in myocardial infarction detection," Computers in Biology and Medicine, vol. 61, 2015, pp. 178-184.

[33] C.A. Bustamante, S.I. Duque, A. Orozco-Duque, and J. Bustamante, "ECG delineation and ischemic ST-segment detection based in wavelet transform and support vector machines," Pan American Health Care Exchanges, 2013, pp. 1-7.

[34] N. A. Bhaskar, "Performance analysis of support vector machine and neural networks in detection of myocardial infarction," Procedia Computer Science, vol. 46, 2015, pp. 20-30.

[35] E. A. Maharaj and A. M. Alonso, "Discriminant analysis of multivariate time series: Application to diagnosis based on ECG signals," Computational Statistics Data Analysis, vol. 70, 2014, pp. 67-87.

[36] G. Bortolan and I. Christov, "Myocardial infarction and ischemia characterization from T-loop morphology in VCG," Computers in Cardiology, 2001, pp. 633-636.

[37] B. Biswal, "ECG signal analysis using modified S-transform", Healthcare Technology Letters, Vol. 4, 2017, pp. 68-72.

[38] N. Kannathal, U. Rajendra Acharya, P. Joseph, L. C. Min and J. S. Suri, "Analysis of Electrocardiograms", Advances in Cardiac Signal Processing, Vol. 22, 2007, pp. 55-82.

[39] I. Tierala, C. Kjell Nikus, S. Sclarovsky, M. Syvänne, M. Eskola, "Predicting the culprit artery in acute ST-elevation myocardial infarction and introducing a new algorithm to predict infarct-related artery in inferior ST-elevation myocardial infarction: correlation with coronary anatomy in the HAAMU Trial", Journal of Electrocardiology, Vol. 42, 2009, pp. $120-127$.

[40] D. Tomar and S. Agarwal, "Feature Selection based Least Square Twin Support Vector Machine for Diagnosis of Heart Disease", International Journal of Bio-Science and Bio-Technology, Vol.6, No.2, 2014, pp. 69-82.

[41] N. Kumar Dewangan and S.P. Shukla, "ECG Arrhythmia Classification using Discrete Wavelet Transform and Artificial Neural Network," IEEE International Conference On Recent Trends In Electronics Information Communication Technology, 2016, pp. 1946-1950.

[42] A. Ratnaparkhi and R. Ghongade, "A Frame Work for Analysis and Optimization of Multiclass ECG Classifier Based on Rough Set Theory," IEEE International Conference on Advances in Computing, Communications and Informatics, 2014, pp. 2740-2744.

[43] Chang-Sik Son, Yoon-Nyun Kim, Hyung-Seop Kim, Hyoung-Seob Park and Min-Soo Kim, "Decision-making model for early diagnosis of congestive heart failure using rough set and decision tree approaches," Journal of Biomedical Informatics, Vol. 45, 2012, pp. 999-1008.

[44] D. A. Orrego, M.A. Becerra, and E. Delgado-Trejos, "Dimensionality reduction based on fuzzy rough sets oriented to ischemia detection," Annual International Conference of the IEEE Engineering in Medicine and Biology Society, 2012, pp. 5282-5285.

[45] A. Kumar and M. Singh, "Ischemia detection using isoelectric energy function," Computers in Biology and Medicine, Vol. 68, 2016, pp. 76-83.

[46] R. O. Duda, P. E. Hart, and D. G. Stork, "Pattern classification," NewYork, NY, USA: Wiley, 2012.

[47] C. K. Roopa and B. S. Harish, "A Survey on various Machine Learning Approaches for ECG Analysis," International Journal of Computer Applications, Vol. 163, 2017. 


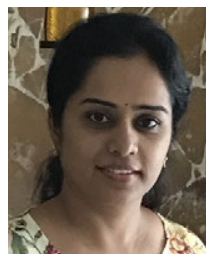

C. K. Roopa

She received her B.E degree in Information Science and Engineering and M.Tech degree in Computer Engineering from Visvesvaraya Technological University, Belagavi, Karnataka, India. She is currently working as a Assistant Professor at JSS Science \& Technology University. She is also pursuing her Ph.D degree in Computer Science from University of Mysore, Karnataka, India. Her area of research includes Machine Learning and Medical Informatics.

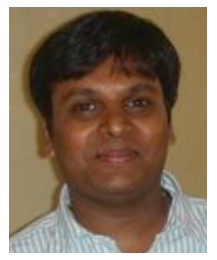

\section{B. S. Harish}

He obtained his Ph.D. in Computer Science (2011) from University of Mysore. Presently he is working as an Associate Professor in the Department of Information Science \& Engineering, JSS Science \& Technology University, Mysuru. He was a Visiting Researcher at DIBRIS - Department of Informatics, Bio Engineering, Robotics and System Engineering, University of Genova, Italy. He has invited as a resource person to deliver various technical talks on Data Mining, Image Processing, Pattern Recognition, Soft Computing. He is also serving and served as a reviewer for National, International Conferences and Journals. He has published articles in more than 60 International reputed peer reviewed journals and conferences proceedings. He successfully executed AICTE-RPS project which was sanctioned by AICTE, Government of India. His area of interest includes Machine Learning, Text Mining and Computational Intelligence. 